\title{
USE OF KODAK TECH-PAN FILM AT THE UKSTU
}

\author{
Q.A. PARKER ${ }^{1}$, S. PHILLIPPS ${ }^{2}$, D.H. MORGAN ${ }^{3}$, D.F. MALIN ${ }^{1}$, K.S. RUSSELL ${ }^{1}$, \\ M. HARTLEY ${ }^{1}$ and A. SAVAGE ${ }^{1}$ \\ ${ }^{1}$ Anglo-Australian Observatory, PO Box 296, Epping NSW 2121, Australia \\ ${ }^{2}$ Department of Physics and Astronomy, University of Wales College of Cardiff, \\ Wales \\ ${ }^{3}$ Royal Observatory, Blackford Hill, Edinburgh EH9 3HJ, Scotland
}

\section{Introduction}

Kodak Technical Pan (Tech Pan) emulsion is an extremely fine grained, high resolution, panchromatic negative film with extended red sensitivity. It has been produced under this name since about 1980 (Kodak P-255, 1981) and is available on Kodak's Estar base in a number of thicknesses and sizes. The thick **base Tech Pan is designated 4415 and has been used with great success by the amateur astronomical community for many years (e.g. Martys 1991). Its astronomical potential was recognised early by Everhart (1981). However, tests at professional telescopes (e.g. West et al. 1981) and early sensitometer tests at the UK Schmidt Telescope (UKST) in 1981 and 1987 were discontinued when the glass and film samples did not respond well to normal hypersensitisation techniques. These and other difficulties led to a lack of interest among the professional astronomical community until quite recently (Russell et al. 1992; Parker \& Malin 1992). The first successful use of $14 \times 14$ inch hypered Tech-Pan 4415 film in the UKST was in March 1991. Films were obtained which exhibited excellent image quality and resolution. Furthermore, in good seeing these appeared to be about 1 magnitude deeper than the equivalent IIIa-F emulsion on glass but with considerably lower grain noise. This result was achieved because two main problems associated with Tech-Pan and film use in the UKST have been resolved. These were:

1) obtaining Tech-Pan film with long exposure speed sufficient for deep astronomical photography (i.e. reduction of low intenstiy reciprocity failure);

2) overcoming the practical difficulties of mounting large-format flexible film at the UKST's curver focal surface.

Fuller details of how this was achieved are given in Parker (1992) and Parker, Malin \& Russell (in preparation). Tech-Pan now accounts for more than $60 \%$ of all non-survey exposures on the telescope and over 250 film exposures have been taken since January 1992, amounting to about 20\% of all current UKST exposures. An exciting variety of different astronomical projects which take full advantage of the superb imaging, low noise and fainter limits offered by Tech-Pan are underway.

Here we present some quantitative results from COSMOS measuring machine scans of TechPan film which clearly demonstrate the information gains over the traditional IIIa-F glass-based emulsion. These improvements lead directly to more effective and efficient use of the UKST and increased scientific productivity. Some of the areas in which Tech-Pan film can make a useful 
scientific impact in Schmidt photography are also described. Coupled with the advent of the new generation of fast, accurate measuring machine 'SuperCOSMOS', and the possibility of digitally co-adding multi-film exposures of the same field for even greater depth, a new lease of life for photographic wide-field imaging is promised.

\section{Basic Properties and First Results at the UKST}

Kodak publications P-255 (1981) and P-315 (1987) detail Tech-Pan's basic properties. It has extremely fine, mono-disperse grain structure leading to low granularity, which is a factor of two better than that for IIIa emulsions. The resolving power is also high, 320 lines/mm, compared with 200 lines/mm for IIIa emulsions. The spectral sensitivity of Tech Pan is similar to that of IIIa-F but is generally flatter, except for a useful enhancement around $H \alpha$. Both the modulation transfer function (MTF) and point spread functions (PSF) are superior to IIIa-F, and the film is capable of recording a wide contrast range, dependent on treatment (Kodak 1987). Finally TechPan's emulsion thickness on Estar is only about $11 \mu \mathrm{m}$ compared with about $20 \mu \mathrm{m}$ for IIIa emulsions, which enhances the other imaging properties mentioned above.

Because of the overall similarity in spectral sensitivity between Tech-Pan and IIIa-F (Kodak 1987), many of the initial comparisons were between these emulsions exposed behind a Schott OG590 filter, used for standard UKST OR-band imaging. After the initial hypersensitising (hypering) tests, 'sky limited' (i.e. developed sky density about 1.0 above chemical fog) Tech-Pan film with the OR-band filter were soon obtained with exposures of about an hour, similar to those required for hypered IIIa-F plates. It was found that the contrast (gamma) of Tech Pan at the sky density was almost identical to IIIa-F. From a sample of 50 Tech Pan films and 50 IIIa-F plates, the gamma at sky density was 2.0 to within $3 \%$. The resultant scientific gains are a direct consequence of the basic properties of the emulsion combined with effective long-exposure speed from appropriate hypering, processing and exposure conditions evolved at UKST (Parker 1992).

\section{Visual assessment comparisons}

Plates 1a and 1b show magnified areas from two consecutive Tech-Pan and IIIa-F UKST exposures (OR14719 and OR14720) taken under similar conditions. In fact the reported seeing for the IIIa-F exposure was superior to that of the preceding Tech-Pan exposure. Both exposures were of 1 hour duration and were taken either side of the meridian. The IIIa-F exposure was subsequently graded to be of 'atlas' or 'survey' quality. Simple visual inspection of this and other sky limited 'OR' Tech-Pan films reveals much improved image resolution compared with the IIIa-F plates even when taken under non-ideal conditions.

The clarity of the stellar images and features in extended objects such as the spiral arms and dust lanes of galaxies was quite remarkable (Russell et al. 1992). Visual assessment of a series of sky-limited Tech-Pan films also indicated a much fainter limit estimated at about a magnitude. The minimum image diameters were also found to be smaller, i.e. 20 - $25 \mu \mathrm{m}$ compared with $30-35 \mu \mathrm{m}$ for III a plates. However, quantitative assessments were required to put these impressions on a much firmer footing. These were obtained by scanning the films with COSMOS, a fast measuring machine (MacGillivray \& Stobie 1984) and were compared with normal IIIa-F data from the same fields. The initial results are discussed by Parker, Morgan \& Phillipps (1993). 


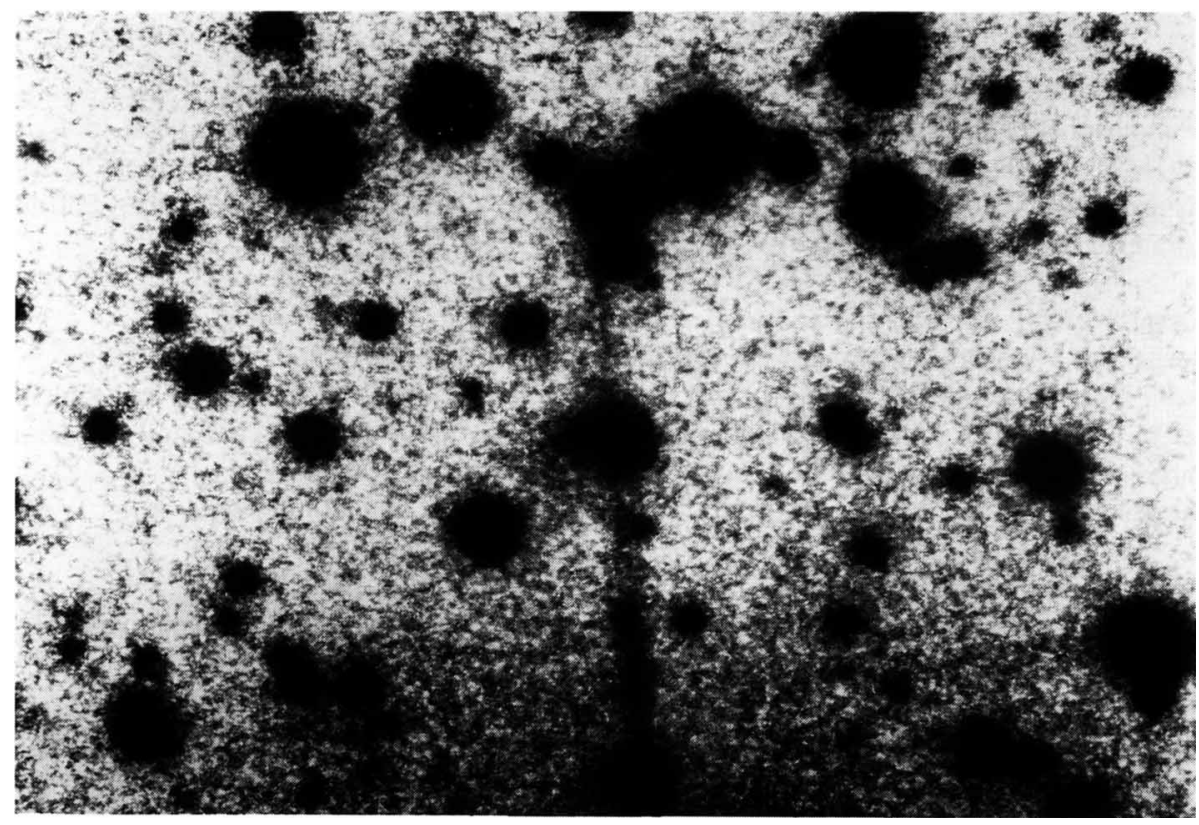

Plate 1a. OR14719 Tech Pan 4415. The prints are approximately $2 \times 1.5$ arcminutes on a side.

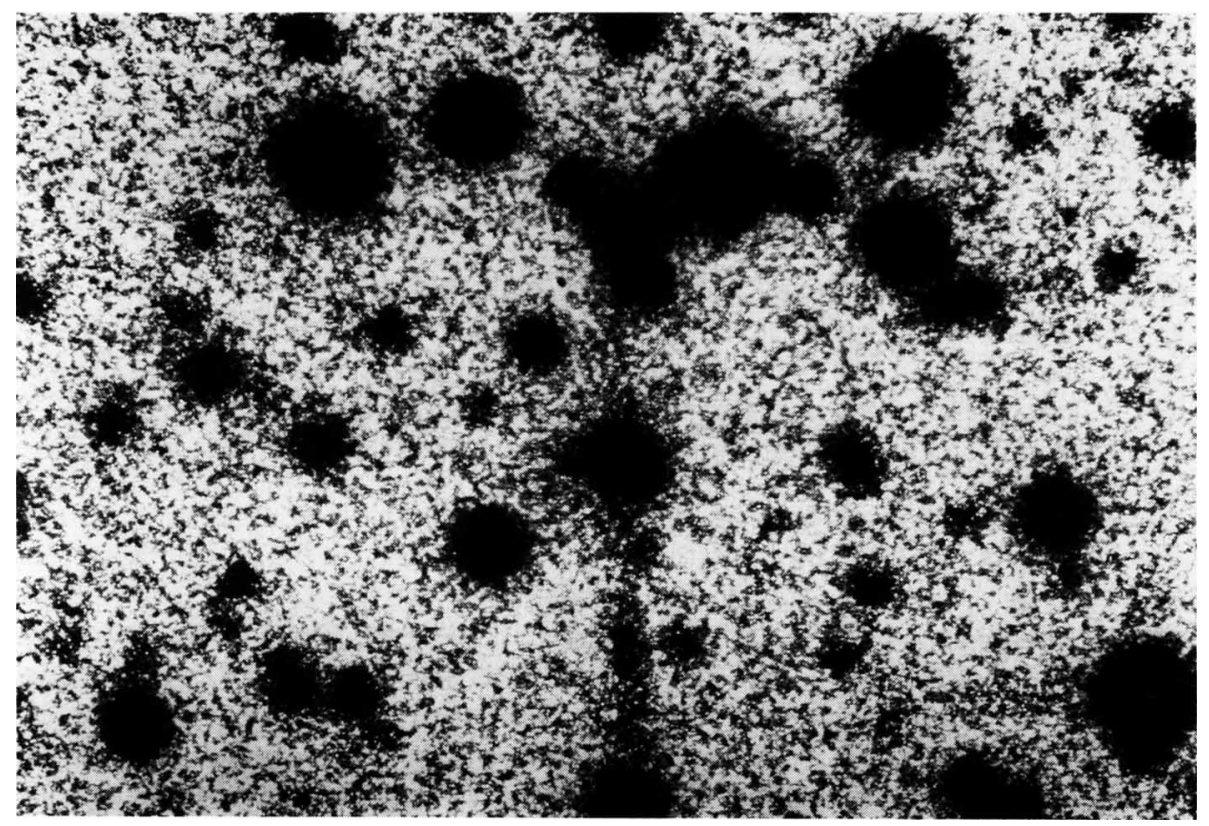

Plate 1b. OR14720 IIIa-F. 


\section{Results from COSMOS Scans of Tech-Pan Films}

To confirm some of the qualitative impressions of the new emulsion, a series of Tech-Pan and IIIa-F exposures of the same fields were scanned with COSMOS. Both direct pixel 'mapping mode' (MM) and thresholded image-analysis mode (IAM) comparisons were made. The basic results are described below, but more detail will appear in a forthcoming paper (Parker, Phillipps \& Morgan, in preparation).

Uniformity of the sky background

Concerns that a very fine grained, high contrast emulsion such as Tech-Pan would be prone to intrinsic, large-scale non-uniformities were not confirmed by the COSMOS data, and the films appear to offer an even more uniform background than the IIIa-F plates. This may be because the large-scale production of Tech-Pan film is not so prone to the batch to batch variations sometimes found in the smaller production runs of glass based IIIa emulsions.

\section{Sky background noise}

Intensity histograms of the sky-background density derived from several film and equivalent IIIaF plate scans indicate a $3-4$ fold reduction in the sky noise to levels around $0.8 \%$ per pixel. Tech-Pan has about 4 times as many grains per unit area compared with IIIa-F, and these grains are about half the size of those in IIIa emulsions, facts which have a direct bearing on the achievable $S / N$ near the sky-background, based on simple Poisson statistics. Figure 1a-b illustrate this property and show intensity histograms from COSMOS MM data from IIIa-F and Tech-Pan UKST exposures of the same area in the VIRGO cluster of galaxies.

\section{Star/Galaxy separation}

Standard COSMOS star/galaxy separation plots such as Log(image-area) versus COSMOS isophotal magnitude reveal much improved star/galaxy separation. The stellar locus is generally extremely tight and well defined for Tech-Pan data allowing discrimination to fainter magnitudes, which should result in improved deep galaxy catalogues. Figures $2 \mathrm{a}$ and $2 \mathrm{~b}$ give examples taken from the field in VIRGO. The plots are restricted to a narrow magnitude range to demonstrate the effect more clearly. More examples are given in Parker, Phillipps \& Morgan (in preparation).

\section{Magnitude limits and detection of Low Surface Brightness features}

Number-magnitude counts from Tech-Pan and IIIa-F COSMOS data of the same fields confirm the visual estimate of a gain of about one magnitude for exposures taken under similar conditions (Phillipps \& Parker, 1992, 1993 and this meeting). The above work has also shown that low surface brightness (LSB) galaxy intensity profiles obtained from COSMOS MM scans of TechPan film can reach $26.5-27.0 \mathrm{R}$ magnitudes per square arcsec (less than $0.4 \%$ of the sky background) and 1 - 2 magnitudes better than for IIIa-F.

\section{General Tech-Pan and IIIa-F image parameter comparisons}

A magnitude plot of paired images from Tech-Pan and IIIa-F COSMOS data of UKST Field 433 is shown in Fig. 3a. The IIIa-F data were from an atlas quality original. Good agreement between the R-band magnitude estimates was found. This appears to be generally true from other comparisons made. The exposure to exposure consistency of Tech-Pan is also well behaved, as shown in Fig. 3b which compares Tech-Pan COSMOS magnitudes from two exposures of the VIRGO field. Other IAM image properties also give good consistency from different Tech-Pan 


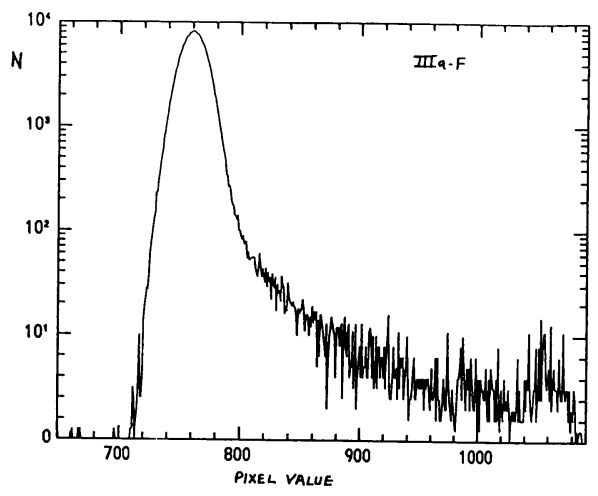

(a)

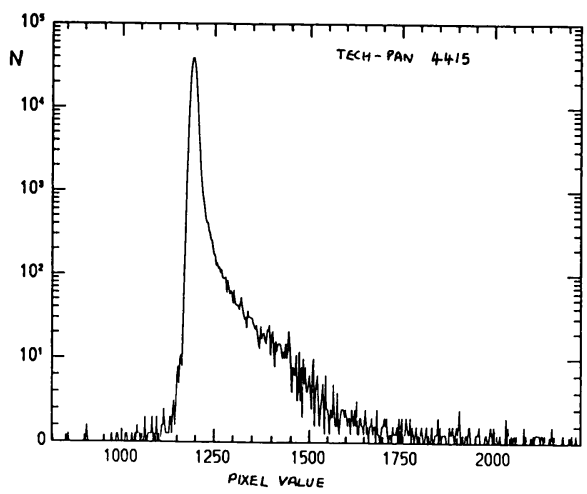

(b)

Figure 1a, 1b. Pixel intensity histograms obtained from COSMOS MM data of the same Virgo field area from IIIa-F and Tech Pan exposures. Note the narrower width of the sky intensity values (as a fraction of the full intensity range in the area) for the Tech Pan data. The pixel sizes for the Tech Pan data were half those of the IIIa-F data and yet the IIIa-F sky noise (as given by the FWHM of the main peak) is about twice that of the Tech-Pan data.

exposures of the same field. An interesting effect is also seen with bright stars, with a bifurcation in the COSMOS stellar magnitude estimates when comparing Tech-Pan and IIIa-F bright star images (see Fig. 3a). This is due to the growth of inner halation rings associated with Tech-Pan exposures, combined with the ability to detect the stellar diffraction spikes to a greater radius from the stellar image centroid against the surrounding background. Images of galaxies are not affected in this way. 


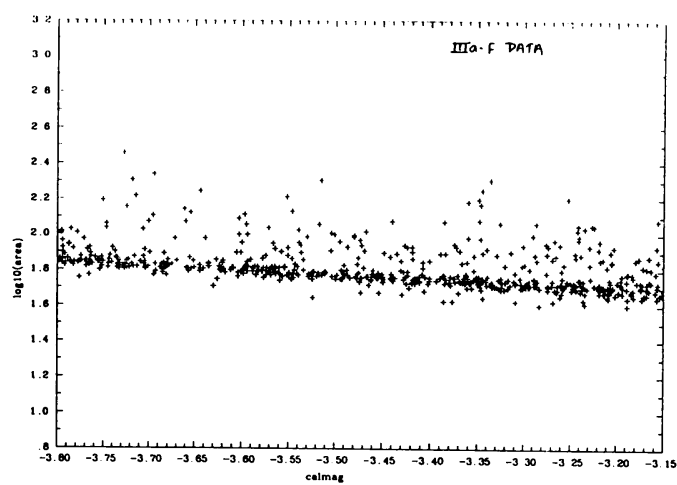

(a)

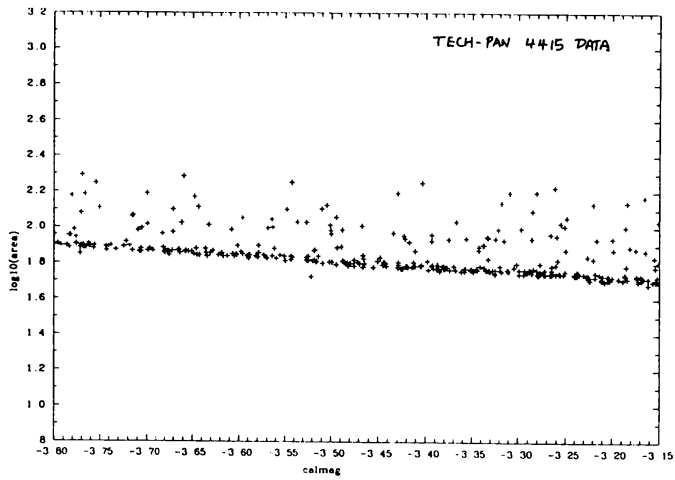

(b)

Figures 2a, 2b. Standard COSMOS star/galaxy separation plots of Log(image-area) versus COSMOS isophotal magnitude estimate (given as magnitude above sky) for IIIa-F and Tech Pan COSMOS IAM data from the same region of the Virgo field. A restricted magnitude range is plotted to better illustrate the tighter nature (and hence better separation) of the stellar locus in the Tech Pan data.

\section{Signal-to-Noise and Detective Quantum Efficiency}

Our first estimates of the detective quantum efficiency (DQE) of the 'optimally' hypered and processed UKST exposures on Tech-Pan film indicate values around 10\% (see Hartley et al., these proceedings). If true this would be a remarkable figure for a photographic emulsion. Our COSMOS data indicate sky background signal-to-noise $(S / N)$ improvements of a factor 3 - 4, allowing the setting of much lower isophotal thresholds.

Figures $4 \mathrm{a}$ and $4 \mathrm{~b}$ show a radial profile through the same faint stellar image on comparable IIIa-F and Tech-Pan COSMOS MM data which illustrates the gain in S/N. This leads directly to fainter magnitude limits and detection of lower surface brightness features. Cannon (1984) argued convineingly for the gains that might be expected with a suitably fast, fine grained emulsion for Schmidt astrophotography. The measurement and detection of images close to the 


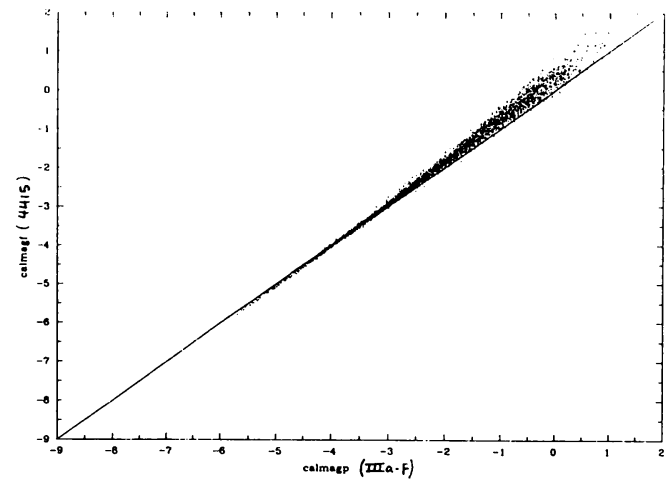

(a)

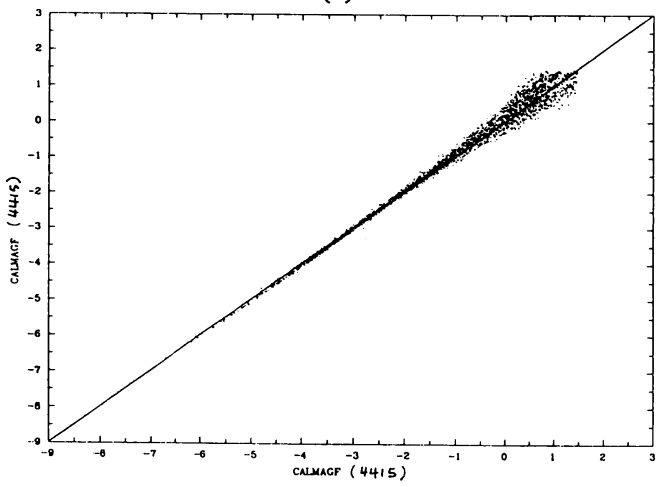

(b)

Figure 3. COSMOS isophotal magnitude plots from paired COSMOS IAM data of Tech Pan and IIIa-F exposures of the same fields. a) Tech Pan versus IIIa-F magnitudes for field 443 . b) Tech Pan magnitude comparisons from two separate exposures of the Virgo field.

plate limit is crucially dependent on the sky-background level and the number of photon events detected. The accuracy with which the brightness or position of a point source image can be obtained on a UKST photograph depends basically on Poisson statistics and thus on the square root of the grain number.

All other things being equal, the number of grains in a stellar image on Tech-Pan is about 4 times higher than for the equivalent image on IIIa-F. This increase in the number of grains will give a factor two improvement in the size of the smallest detected extended objects at a given level of surface brightness (Cannon 1984). This also equates to a gain of 0.75 magnitudes for a given image size. Since we have obtained Tech-Pan exposures which reach the same sky density as IIIa-F exposures in the same exposure time, we estimate that the DQE of optimally hypered Tech-Pan is increased by a factor of 4 relative to similarly hypered IIIa-F. 


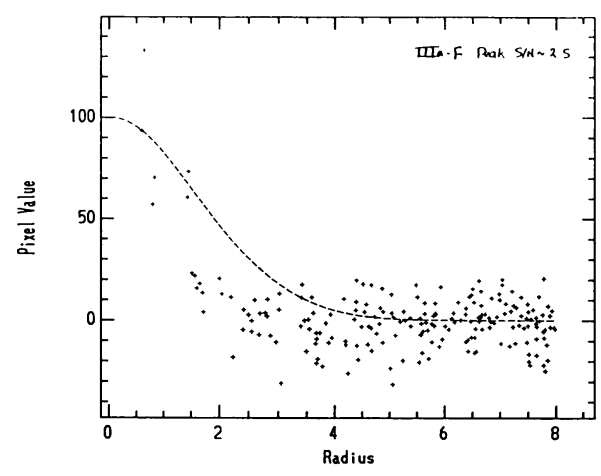

(a)

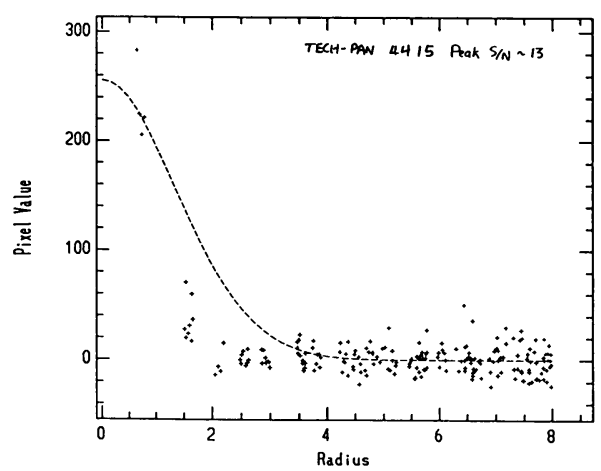

(b)

Figures 4a, 4b. Radial profiles taken through COSMOS MM data of a region centred on the same faint star from a) IIIa-F and b) Tech Pan exposures of the Virgo field. Note the significant improvement in peak S/N for the Tech Pan data.

\section{Astrometry from original Tech-Pan film exposures}

An important concern with original Tech-Pan film exposures is their ability to produce good relative astrometry. Initial studies indicate no apparent loss in astrometric fidelity which can be attributed to Estar film. Kodak leaflet Q-34 (Kodak 1970), shows their Estar base is extremely stable, with excellent strength and thermal stability, which is only 1.8 times worse than glass plates (i.e. only $0.001 \%$ size change per ${ }^{\circ} \mathrm{F}$ ). Van Haarlem et al. (1992) have also shown that film copy material on a similar substrate can achieve essentially the same astrometric fidelity as original glass plates. However, unlike copy film, original film exposures are subjected to deformations at the UKST's $3 \mathrm{~m}$ radius focal surface and changes in humidity and temperature during exposure. Concerns have been expressed about the ability of the material to mechanically recover from deformation and yield good astrometry.

Our COSMOS data from paired IIIa-F and Tech-Pan exposures of the same fields gave good 
agreement between co-ordinates to better than 0.5 arcseconds $(7 \mu \mathrm{m})$, obtained from fits to large numbers of standards in each field. Paired Tech-Pan exposures of the same field gave even better agreement (about 0.3 arcseconds). If non-uniform stretching was a serious problem such good results (typical of the general accuracy of COSMOS data) would not have been obtained.

Finally, Evans (1993) has used Automatic Plate Measuring machine (APM) data and a more stringent astrometric evaluation of Tech-Pan film. Preliminary results indicate that glass based IIIa emulsions and Tech-Pan provide essentially the same accuracy for relative astrometry. Repeat measures of the positions of stars measured from Tech-Pan and IIIa-F exposures scanned $180^{\circ}$ apart gave $\sigma($ Tech-Pan $)$ of $0.04 \mathrm{arcsec}$ and $\sigma(I I I a-F)$ of 0.05 arcsec. Further studies on a larger set of exposures are underway (Evans, in preparation).

\section{Current Uses of Tech Pan at the UKST and Future Projects}

The preliminary results described above indicate that Tech-Pan has many potential uses at the UKST, largely as a direct and superior replacement for IIIa-F R- and OR-band imaging for nonsurvey and, perhaps, survey type work. It could also be used as a replacement for U-band imaging for which IIIa-J is currently used. Though the U filter has a 'red leak', it does not appear to be significant (Parker, Morgan \& Phillipps 1993). Finally one of the obvious benefits of film use is as an inexpensive means of providing follow-up exposures for observations of transient phenomena, comets, fast moving asteroids, novae, etc. New projects on large Schmidt telescopes with Tech-Pan will exploit its excellent imaging, high resolution and very low noise characteristics.

\section{Objective prism work}

Tech-Pan is an effective alternative to IIIa-F objective-prism exposures. The spectral sensitivity curve is flatter, which means that Tech-Pan should be much better for high redshift QSO searches, where the bumps in the IIIa-F spectral sensitivity can mimic emission lines. Preliminary results (Drinkwater 1993, AAO Newsletter No. 64) indicate that the improved resolution and S/N do enable better feature discrimination. Objective-prism searches of the Magellanic clouds for planetary nebulae, carbon stars etc. would also benefit from the greater $\mathbf{S} / \mathrm{N}$ and resolution of Tech-Pan.

\section{H $\alpha$ survey work}

The enhanced sensitivity peak of Tech-Pan around H $\alpha$ suggest that it could replace the 098 and III-F emulsions for $\mathrm{H} \alpha$ narrow-band imaging. A recent 3 hour $\mathrm{H} \alpha$ film went almost as deep as the ESO R survey. New H $\alpha$ survey work in selected regions to take advantage of the sensitivity peak and better resolution should be considered.

\section{Deep survey work from Tech-Pan Film co-addition}

This can be accomplished both photographically (Malin 1981) or digitally (Marston 1988; Hawkins 1992). Digital co-addition exciting potential and allows greater control, manipulation and quantification than photography, though is a good deal more time consuming. Full field addition is now much more practical due to the availability of cheap mass storage media and faster computers to account for rotation, translation and scale problems that limit photographic addition. OR exposures on Tech Pan are already a magnitude fainter than existing $R$ survey material which has an approximate limiting $R$ magnitude of 22 . The co-addition of 6 films of 
the same field would gain a further magnitude, reaching 24th magnitude in $R$. With the next generation measuring machines such as SuperCOSMOS soon to be available (Miller 1992), this technique could be a cheap, cost-effective alternative to an UKST CCD mosaic for performing deep surveys. Work on faint galaxy number-counts, galaxy clusters, stellar astrometric and evolutionary studies over very large scales become possible.

\section{Wide area searches for Low Surface Brightness (LSB) Galaxies}

The evident excellent capability of Tech-Pan for detecting extended LSB features should enable effective searches for LSB galaxies over large areas, especially if digital co-addition was also employed. A number of projects in this vein are already underway with the UKST.

\section{Multi-colour work}

Reasonable approximations to the standard $U$ and $R$ passbands can be obtained using Tech-Pan and suitable filters, allowing $U$ - R measurements. Narrow band $H \alpha$ imaging is also practical. Although it is possible to isolate approximations to B and V colours with Tech-Pan, the emulsion sensitivity in this region is not particularly high and there are potential problems with the redleakage common to most UV filters. For B and V imaging, a IIIa-J or blue sensitive version of Tech-Pan would be extremely valuable. Apart from a direct replacement for IIIa-J B-band exposures, the B sensitisation would be useful in a wide variety of multi-colour projects, including UBVR Quasar searches.

\section{The Main Advantages and Disadvantages of Tech-Pan}

\section{Practical advantages}

A sheet of Tech-Pan film costs one tenth as much as an equivalent glass plate, and offers obvious transportation, storage and handling benefits. The Estar-base itself is extremely stable, having excellent strength, toughness and flexibility properties (Kodak publication Q-34, 1977). Since Tech Pan is produced in large quantities, it does not suffer the same batch to batch variations that plague Kodak spectroscopic emulsions on glass. Finally, the hypered product stores extremely well in an inert atmosphere in cold storage with no apparent degradation over a month or more.

\section{Current known disadvantages}

Tech-Pan is proving difficult to copy and needs careful mounting for proper scanning with a measuring machine such as COSMOS. Film is also more prone to kinking and to static and tends to pick up dust easily. Longer development times may be required for optimum detectivity but this is not a serious problem, especially since film lends itself to machine processing. Finally, more halation effects are seen with bright images than with IIIa-F.

\section{Conclusions}

The introduction of Tech-Pan film at the UKST has led directly to considerable scientific and economic benefits. The improved depth, resolution and noise characteristics of Tech-Pan has offered a new and exciting lease of life to deep, wide-field astrophotography at the UKST. 


\section{References}

Cannon, R.D., 1984. In 'Astronomical Photography', Occasional Reports of the Royal Observatory Edinburgh, eds. M.E. Sim and K. Ishida, p. 119.

Evans, D.W., 1993. IAU Commission No. 9, Working Group on Wide-field Imaging, Newsletter No. 3, p. 64 .

Everhart, E., 1981. In 'Astronomical Photography', Occasional Reports of the Royal Observatory Edinburgh, eds. J.L. Heudier and M.E. Sim, p. 117.

van Haarlem, M.P., le Poole, R.S., Katgert, P. and Tritton, S., 1992. Mon. Not. R. astron. Soc., 255, 295.

Hawkins, M.R.S., 1992. IAU Commission No. 9, Working Group on Wide-field Imaging, Newsletter No. 1, p. 23.

Kodak Publication Q 34, 1970. 'Dimensional Stability of KODAK Estar Base Films for the Graphic Arts'.

Kodak Publication P-255, 1981. 'KODAK Technical Pan Film'.

Kodak Publication P-315, 1987. 'Scientific Imaging with KODAK films and plates'.

MacGillivray, H.T. and Stobie, R.S., 1984. Vistas in Astronomy, 27, 433.

Malin, D.F., 1981. J. Photogr. Sci., 21, 199.

Marston, A.P., 1988. Mon. Not. R. astron. Soc., 255, 295.

Martys, C.R., 1991. J. Br. Astron. Assoc., 101, 4.

Miller, L., Cormack, W., Paterson, M.J., Beard, S.M and Lawrence, L., 1992. In 'Digitised Optical Sky Surveys', eds. H.T. MacGillivray and E.B. Thomson, Kluwer Academic Publishers, Dordrecht, p. 133.

Parker, Q.A., 1992. 'Report on Kodak Tech Pan 4415 Estar-based emulsion', AAO internal document.

Parker, Q.A. and Malin, D.F., 1992. 'Recent experience with Kodak Tech-Pan film', in IAU Commission No. 9, Working Group on Wide-field Imaging Newsletter No. 2, p. 24.

Parker, Q.A., Morgan, D.H. and Phillipps, S., 1993. 'Tech Pan UKST films: Some preliminary COSMOS data comparisons', in IAU Commission No. 9, Working Group on Wide-field Imaging Newsletter No. 3, p. 60.

Phillipps, S. and Parker, Q.A., 1992. 'Photometry with Estar film', in IAU Commission No. 9, Working Group on Wide-field Imaging Newsletter No. 1, p. 29.

Phillipps, S. and Parker, Q.A., 1993. 'Galaxy surface Photometry with Kodak Tech Pan film', Mon. Not. R. astron. Soc., 265, 385.

Russell, K.S., Malin, D.F., Savage, A., Hartley, M. and Parker, Q.A., 1992. 'The use of Eastman Kodak 4415 film in the UKST', in 'Digitised Optical Sky Surveys', eds. H.T. MacGillivray and E.B. Thomson, Kluwer Academic Publishers, Dordrecht, p. 23.

West, R.M., Kurtanidze, O.M., Geonijan, L.A. and Kimeridze, G.N., 1981. AAS Photo-Bulletin, No. 28 , p. 3. 